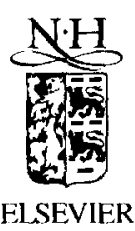

\title{
A generalized streamline finite element approach for the analysis of incompressible flow problems including moving surfaces
}

\author{
Marcela A. Cruchaga*, Eugenio Oñate \\ International Center for Numerical Methods in Engineering, E.T.S. d'Enginyers de Camins, Canals $i$ Ports, \\ Universitat Politècnica de Catalunya, Gran Gapità s/n, Mòdul C1, 08034 Barcelona, Spain \\ Received 18 February 1997; revised 4 February 1998
}

\begin{abstract}
In the present work a generalized streamline finite element formulation able to deal with incompressible flow problems is presented. In the finite element framework, this technique allows the use of equal order interpolation for the unknowns of the problem: velocity and pressure. In this context, stable and convergent solutions can be obtained without requiring tuning parameters defined outside this model. The tracking of moving surfaces is also included in the numerical model. This formulation has been checked in 2D and 3D tests. (C) 1999 Elsevier Science S.A. All rights reserved.
\end{abstract}

\section{Introduction}

In this paper, a new generalized streamline operator (GSO) technique is used in the numerical solution of the incompressible Navier-Stokes equations in the framework of the finite element method [1-3]. Several authors have developed recent formulations to overcome the oscillations in the numerical solution when the convective term becomes relevant [4-12]. As is well known, the GSO type technique circumvents the classical mathematical requirements imposed over the choice of the discrete approximation functions (BB conditions) [4-12]. In particular, equal order interpolation functions can be used in the discretization of the primitive variables of the problem: velocity and pressure. Moreover, the standard penalization methods necessary to fulfil the incompressibility equation are not required. In this context, the choice of the upwinding parameters is crucial in order to obtain stable and convergent formulations [1-2]. In the present work an upwinding tensor, formulated and extensively discussed in [1-4], is used. It is important to note that this upwinding tensor does not require tuning parameters defined outside this model and it satisifies the design conditions defined by Hughes et al. [4,3].

The aim of this work is to check more extensively the GSO formulation for incompressible flow problems with particular emphasis in the expressions of the finite element matrices involved and the analysis of 3D and moving surface problems.

The governing equations for the incompressible flow problem written as a generalized convection-diffusion system are briefly described in Section 2. In Section 3, the weak form and the finite element formulation are presented. The SG (standard Galerkin) and GSO contributions in all the matrices and vectors involved in the formulation can clearly be distinguished.

In Section 4, several numerical examples are presented. The driven cavity flow problem is analysed in 3D and a comparative analysis with other numerical techniques is performed. In order to check the moving surface

\footnotetext{
* Corresponding author.
} 
tracking algorithm [13], a two-liquid interface problem is solved in 3D using the presented methodology. Finally, the propagation of a solitary wave and a sloshing problem are also analyzed.

\section{Governing equations}

The basic formulation for incompressible flow problems considering a Newtonian fluid are described by the Cauchy's equation of motion and the continuity equation. These equations can be written as a generalized convection-diffusion system $[1-4,14]$. In this way, the formal problem consists of finding $U$ verifying this system of equations such that:

$$
\Re(\boldsymbol{U}) \equiv \boldsymbol{M} \cdot \dot{U}+\boldsymbol{A}: \boldsymbol{L}-\boldsymbol{\nabla} \cdot(\boldsymbol{K}: \boldsymbol{L})-\boldsymbol{F}=\mathbf{0} \quad \text { in } \Omega \times[0, T]
$$

subject to appropriate boundary and initial conditions, traditionally defined as [14]:

$$
\begin{array}{ll}
\boldsymbol{U}=\left(\bar{u}_{1}, \bar{u}_{2}, \bar{u}_{3}, u_{4}\right) & \text { in } \Gamma_{u} \times[0, T] \\
n \cdot(p \mathbb{I}-\mathbb{K}: L)=\bar{t} & \text { in } \Gamma_{\sigma} \times[0, T] \\
U_{0}=U(x, 0) & \text { in } \Omega
\end{array}
$$

where standard notation is used, $\Omega$ is an arbitrary open bounded domain with smooth boundary $\Gamma,[0, T]$ is the time interval of interest, $\boldsymbol{I}$ is the Kronecker tensor, $\bar{t}$ is the traction vector, $\boldsymbol{n}$ is the outward unit vector normal to the boundary $\Gamma$ such that $\Gamma_{u}$ and $\Gamma_{\sigma}$ are the parts of $\Gamma$ where the velocity and the traction forces are prescribed respectively $\left(\Gamma \cup \Gamma=\Gamma\right.$ and $\left.\Gamma \cap \Gamma_{\sigma}=\emptyset\right)$. Besides, $U$ is the vecter of unknowns $U=\left[u_{1}, u_{2}, u_{3}, u_{4}\right]$ where $\boldsymbol{m}_{1}$

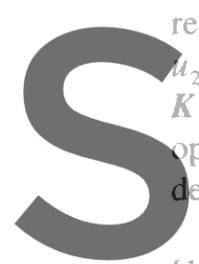

$t_{2}$ and $u_{3}$ are the velocity $K=K_{\text {jimn }}$ is a generalized diffusion

perator; $M$ is the generalize

etails).

Other possibilities in the choice of thes

the

components separately, i.e.
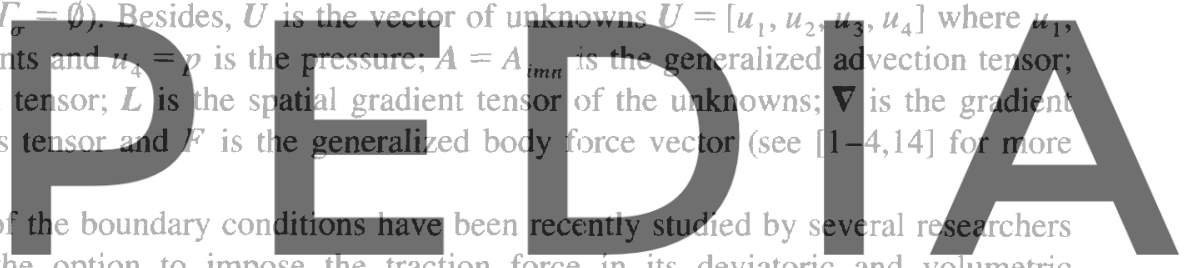

and deviatoric and volumetric $\mathbb{U}=\left(u_{1}, u_{2}, u_{3}, \bar{u}_{4}\right)$ in $\Gamma_{p} \times[\mathcal{O}, T]$

$$
\boldsymbol{n} \cdot(\boldsymbol{K}: \boldsymbol{L})=\boldsymbol{H} \text { in } \boldsymbol{\Gamma}_{H} \times[0, T]
$$

where $\boldsymbol{H}$ is the prescribed traction due to the deviatoric stress tensor. Besides, $\Gamma_{p}, \Gamma_{H}$ are the parts of $\Gamma$ on which the pressure and the deviatoric part of the stress tensor are prescribed, respectively. The intersection between $\Gamma_{u}$, $\Gamma_{p}$ and $\Gamma_{H}$ is an empty set and its union is the whole boundary $\Gamma$ [15-18].

\section{Weak form and finite element formulation}

The variatonal form of the problem expressed by Eqs. (1) and (2) is assumed to be [1-4]:

$$
\int_{\Omega} \boldsymbol{\Psi} \cdot \Re(\boldsymbol{U}) \mathrm{d} \Omega+\int_{\Omega} \boldsymbol{P}(\boldsymbol{\Psi}) \cdot \Re(\boldsymbol{U}) \mathrm{d} \boldsymbol{\Omega}+\int_{\Gamma_{H}} \boldsymbol{\Psi} \cdot[\boldsymbol{n} \cdot(\boldsymbol{K}: \boldsymbol{L}(\boldsymbol{U}))-\boldsymbol{H}] \mathrm{d} \Gamma=0 \quad \forall \boldsymbol{\forall} \in \Theta
$$

where $\Theta$ is the space of the weigthing funtions chosen in agreement with the space of the unknowns $U[4,14]$. $\boldsymbol{P}(\boldsymbol{\Psi})$ is the perturbation function added to the standard Galerkin weighting function is defined as in [1-4].

In the framework of the finite element method [14], it is assumed that the continuous field of the unknowns is locally approximated by polinomial functions in the standard manner as [14]:

$$
{ }_{n} \boldsymbol{U}=\boldsymbol{N}^{k}(\boldsymbol{X}, t) \hat{\boldsymbol{U}}^{k} \quad k=1, \ldots, n_{\text {node }}
$$

where ${ }_{h} U$ is the approximation of the continuous vector of the unknowns $U, \hat{U}^{k}$ is the vector of nodal unknowns 
associated to the node $k$ and $N^{k}$ is the typical shape function matrix for the nocle $k$ used in the standard finite element formulations written as (3D case) [14]:

$$
\boldsymbol{N}^{k}=\left[\begin{array}{cccc}
N_{1}^{k} & 0 & \cdot & \cdot \\
0 & N_{2}^{k} & \cdot & \cdot \\
0 & 0 & N_{3}^{k} & \cdot \\
\cdot & \cdot & \cdot & N_{4}^{k}
\end{array}\right]
$$

where the shape functions $N_{i}^{k}$ are calculated in the spatial point of interest $\left(k=1, \ldots, n_{\text {node }}\right)$ and $i$ indicates the variable to be interpolated $(i=1, \ldots, 1+n$ dim).

Considering that Eq. (3) could be satisfied for any $\Psi$, each function belonging to the canonical base of the discrete space of the unknowns are chosen in order to verify that expression [14]. Taking into account Eq. (4), the assembly of the elemental algebraic system of equations can be written as [3]:

$$
\boldsymbol{R}(\hat{U}) \equiv \mathscr{M} \hat{U}+K \hat{U}-F(\hat{U})-\ddot{H}=0 .
$$

The well-known element matrices for the standard Galerkin method (SG) are described in Box 1. The new terms obtained from the proposed generalized streamline operator methodology (GSO) are shown in Box 2. The total element matrices used in the definition of Eq. (6) are presented in Box 3. The boundary conditions are only considered in the SG formulation $[4,5,14]$. It should be noted, from Box 3, that the matrix $K$ is defined considering different contributions: the convection effects, the diffusion term and the boundary conditions when they are expressed with Eq. (2.4)-(2.5). The GSO technique affects the mass matrix, the $\mathbb{K}$ matrix in its convection and diffusion parts, and the body force vector. These contributions are responsible of the stability and accuracy of the new CSO methedology studied for the incompressibla Navier-Stokes equations. In particular, a very good numerical behavioun can be observed in moderated and high Reynolds's number problems [1-3]. Further, the numerical solution for the Stokes and low Reynolds' numbers problems show also

very good performance $[1-3]$.
The additional notation used in Boxes 1 and 2 are

- $N=\left[\begin{array}{llll}N^{1} & N^{2} & \ldots & N^{n} \text { nde }\end{array}\right]$, where $N^{k}$ con $k=1, \ldots, n_{\text {node }}$ as in Eq. (5)

\section{Register for free at https//www.scipedia.com to download the version without the watermark} Box

Element matrices of the standard Galerkin method

Mass matrix

$$
\mu_{\mathrm{SG}}=\int_{\Omega_{e}} N^{3} M N \mathrm{~d} \Omega_{e}
$$

Convection generalized matrix

$$
\boldsymbol{K}_{\mathrm{SG}}^{c}(\hat{\boldsymbol{U}})=\int_{\Omega_{e}} \boldsymbol{N}^{\tilde{J}} \boldsymbol{A}_{n}^{u} \nabla_{n}\left(\boldsymbol{N}^{u}\right) \mathrm{d} \Omega_{e}-\int_{\Omega_{e}} \nabla_{n}(\boldsymbol{N})^{\exists} \boldsymbol{A}_{n}^{p} \boldsymbol{N}^{p} \mathrm{~d} \Omega_{e}
$$

Diffusion generalized matrix

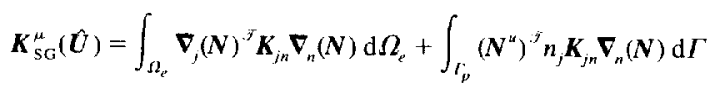

Pressure term matrix

$$
\boldsymbol{K}_{\mathrm{SG}}^{H}=\int_{l_{H}}\left(N^{\mu}\right)^{7} \boldsymbol{n}^{7} N^{D} \mathrm{~d} \Gamma
$$

Generalized vector of body forces

$$
\xi_{\mathrm{sG}}=\int_{\Omega_{e}} N^{-\tau} \mathrm{F} \mathrm{d} \Omega
$$

Generalized vector of imposed surface forces

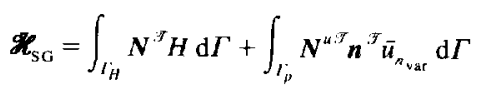


Box 2

Element matrices of the GSO proposed

Mass matrix

$$
\mathscr{M}_{\mathrm{G}, S \mathrm{O}}=\int_{\Omega_{e}} P(N)^{\jmath} M N \mathrm{~d} \Omega_{e}
$$

Generalized convection matrix

$$
\boldsymbol{K}_{\mathrm{G} S O}(\hat{\boldsymbol{U}})=\int_{\Omega_{r}} \boldsymbol{P}(N)^{\prime} \boldsymbol{A}_{n} \boldsymbol{\nabla}_{n}(\boldsymbol{N}) \mathrm{d} \Omega
$$

Generalized diffusion matrix

$$
K_{\mathrm{GSO}}^{\mu}(\hat{U})=-\int_{\Omega_{e}} \boldsymbol{P}(\boldsymbol{N})^{\cdot t} \nabla_{j} \boldsymbol{K}_{j n} \nabla_{n}(\boldsymbol{N}) \mathrm{d} \Omega_{e}
$$

Generalized vector of body forces

$$
F_{\mathrm{GSO}}=\int_{\Omega_{s}} \boldsymbol{P}(\boldsymbol{N})^{r} \boldsymbol{F} \mathrm{d} \Omega_{r}
$$

Perturbation function

$P(N)=\pi A_{n} \nabla_{n}(N)$

Box 3

Total element matrix

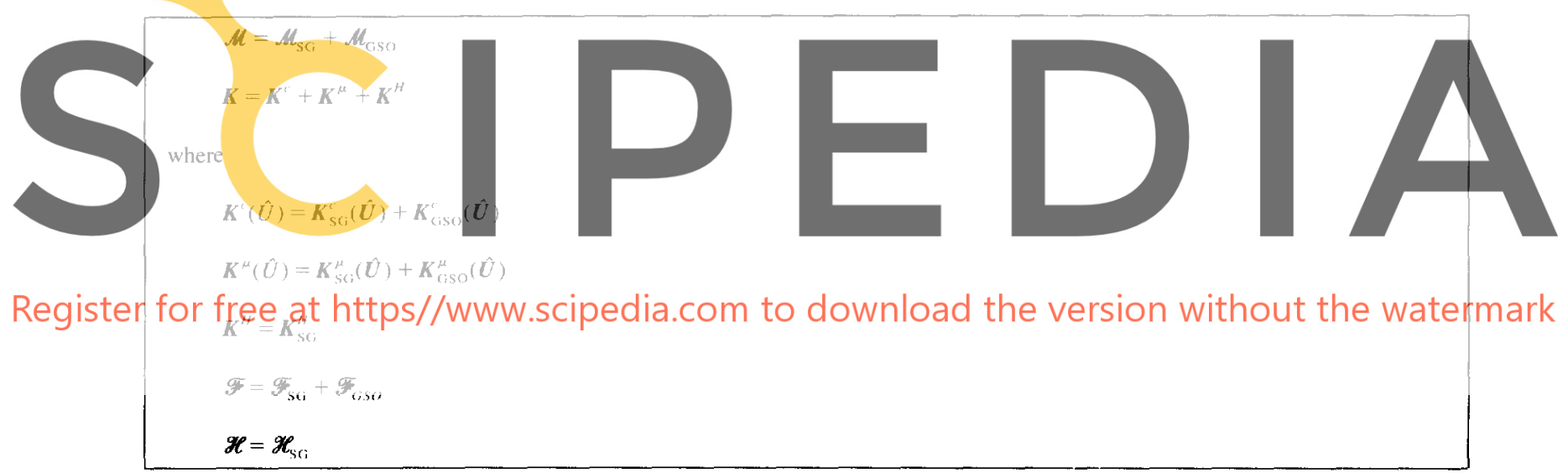

- $N^{\prime \prime}$ are the $N$ previously described in which the shape function associated to the pressure degrees of freedom are neglected.

- $N^{p}$ are the $N$ previously described in which all the shape function are neglected except that associated to the pressure degrees of freedom.

It should be noted that $N^{u}+N^{n}=N$.

- $A_{n}$ are the generalized convection tensor defined for each fixed direction $n$ as in $[1-4 \mid$.

- the $\boldsymbol{A}_{n}^{\prime \prime}$ are the $\boldsymbol{A}_{n}$ where the columns associated with the pressure degrees of freedom are neglected.

- the $\boldsymbol{A}_{n}^{\prime \prime}$ are the $\boldsymbol{A}_{n}$ where all the components are neglected except that corresponding to the pressure degrees of freedom.

It should be noted that $\boldsymbol{A}_{n}^{\prime \prime}+\boldsymbol{A}_{n}^{p}=\boldsymbol{A}_{n}$.

- $\boldsymbol{K}_{j n}$ are the generalized diffusion tensor written for each pair of fixed directions $j n$ [1-4].

- $\nabla_{n}=\partial / \partial x_{n}$.

$-\tau$ is the 'upwinding tensor' [1-3].

A Newton-type incremental-iterative formulation is used for solving the nonlinear semidiscrete system (6) and the convergence criterion is written in terms of the norm of the residual vector where the admissible tolerance is taken in the range of $\left[10^{-10}, 10^{-4}\right]$ throughout this work. 


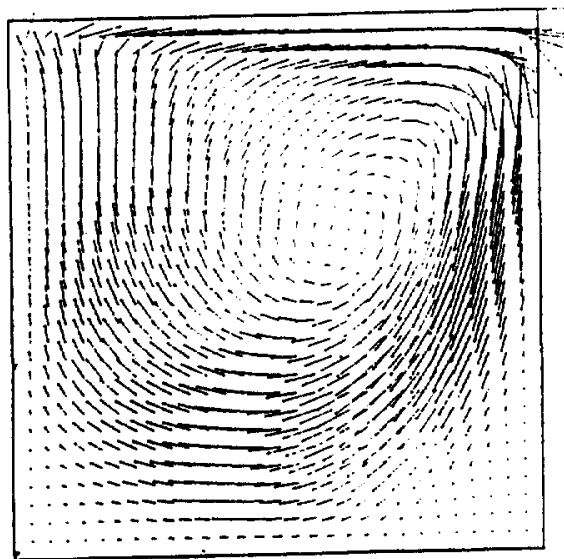

a)

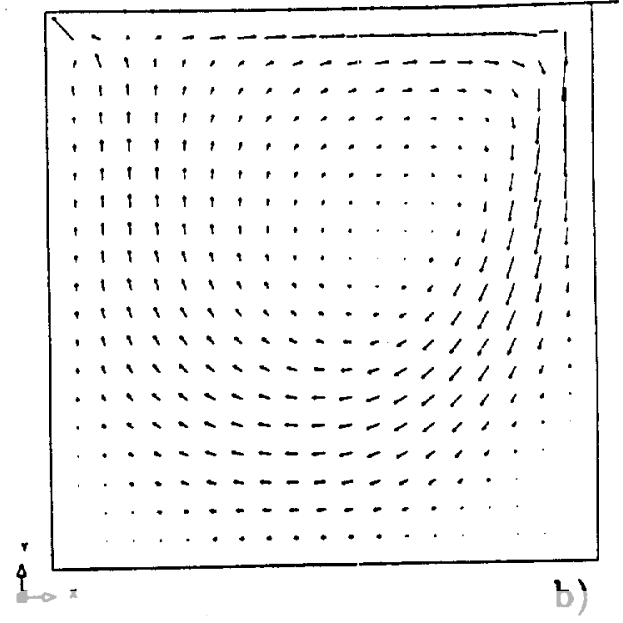

X-Y SYMMETRY PLANE
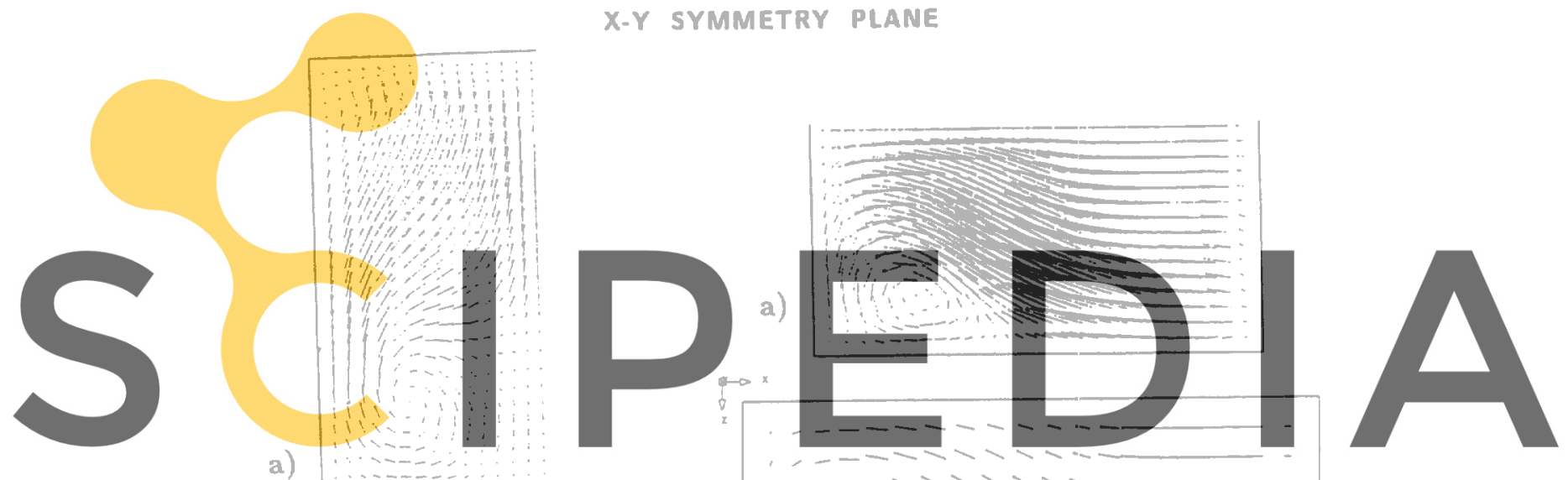

Register for free at https//www.scipedia.com to downito ad the version without the watermark

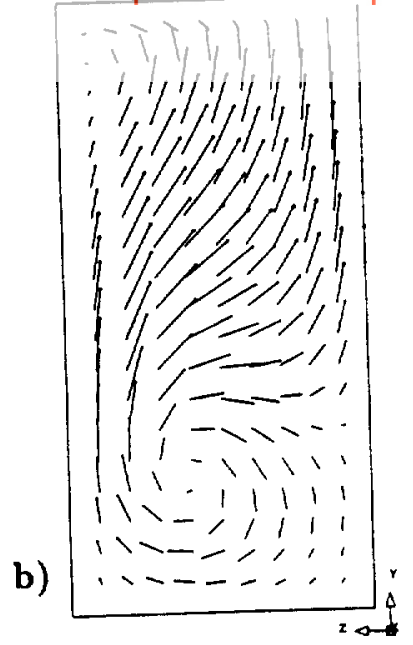

b)

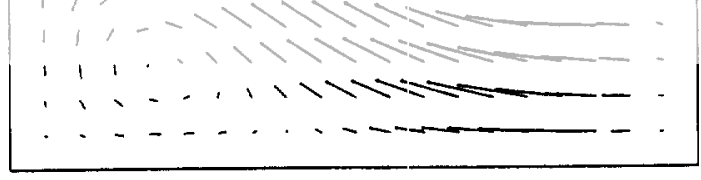

MIOPLANE $y=0.5$

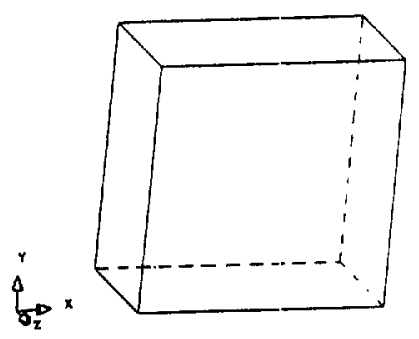

\section{MIDPLANE $x=0.5$}

Fig. 1. 3D cavity flow problem at $\mathrm{Re}=400$. Velocity vectors (a) Guj et al. [19]: (b) present work. 


\section{Numerical examples}

\subsection{Cavity flow problem in $3 D$}

The behaviour of this methodology is checked in a 3D analysis. To this end, a 3D cavity flow problem at $\mathrm{Re}=400$ described by Guj et al. [19] is analyzed. The geometry is a cubic domain of unitary side. The velocity are prescribed to zero in the walls. In the upper face the veloicty field takes the values $U x=1.0, U y=0.0$ and $U z=0.0$. In the edges of this upper face the velocity is fixed to $U x=0.0$. Due to symmetry considerations in the third direction, the analysis is performed over a half domain. A regular mesh with $20 \times 20 \times 10$ eight-noded isoparametric elements in the $x, y$ and $z$ directions are used for the computation. The initial value for the

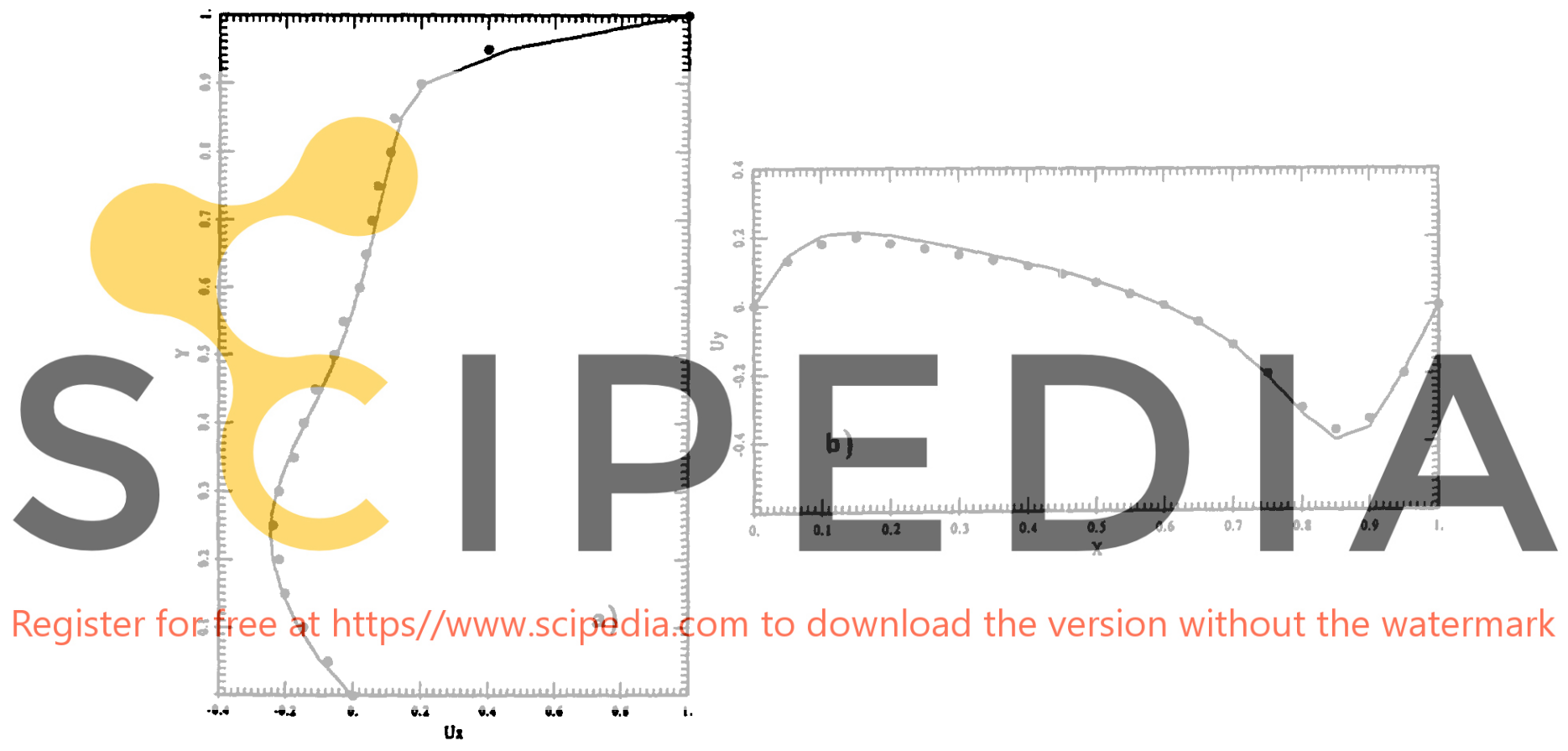

Fig. 2. 3D cavity flow problem at $\mathrm{Re}=400$. (a) Horizontal component of the velocity at $x=0.5$ of the $X-Y$ plane; (b) vertical component of the velocity at $y=0.5$ of the $X-Y$ plane. $\bullet$ Guj et al. [19].-Present work.

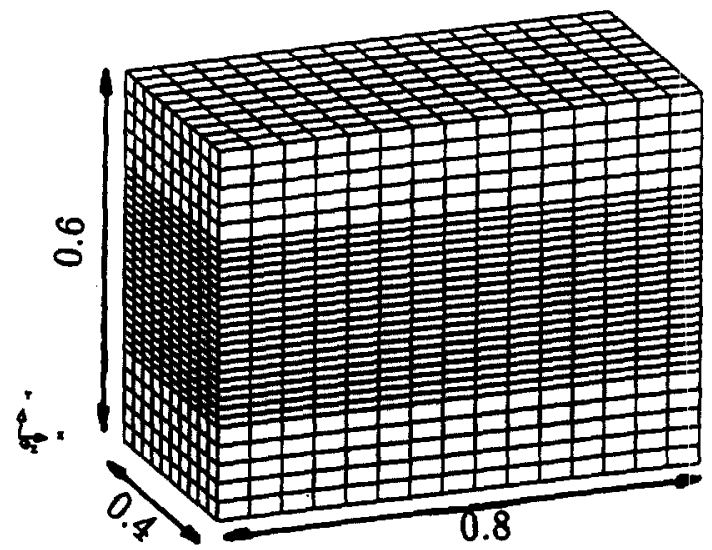

Fig. 3. Two-liquid interface problem in 3D. Geometry and finite element mesh. 
velocity and pressure fields are zero in the whole domain and a steady state analysis is performed. In Fig. 1 the velocity vectors are plotted in the symmetry plane $X-Y$ and the midplanes $x=0.5$ and $y=0.5$. The results are in good agreement with those reported in [19] with a mesh of $67 \times 67 \times 27$ points (also with symmetry conditions). The $x$ and $y$ velocity components are shown in Fig. 2 along the lines $x=0.5$ and $y=0.5$ belonging to the $X-Y$ plane. In this figure the results are compared with the solutions presented in [19]. The profiles show a very good agreement with the velocity contours published by Guj et al. [19].
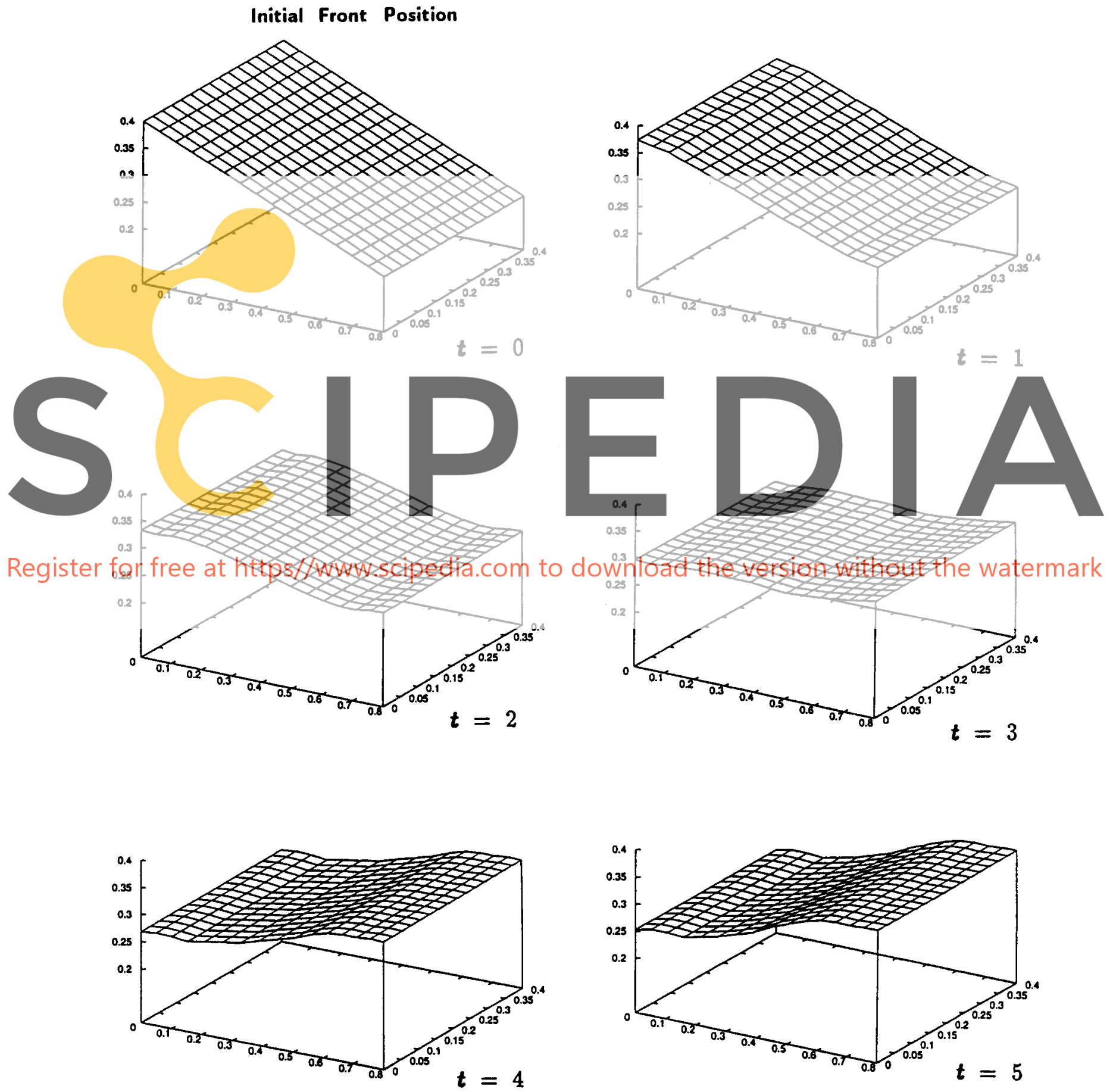

Fig. 4. Two-liquid interface problem in 3D. Interface position at diffent time steps. 


\subsection{Two-liquid interface problem in $3 D$}

This problem is the 3D extension of the two-liquid interface problem in $2 \mathrm{D}$ analyzed in $[2,3,20]$. Two liquids with the same dynamic viscosity and different densities equal to 1.0 and 2.0, respectively, occupy a closed tank with dimensions $0.8 \times 0.6 \times 0.4$ (see Fig. 3). The initial interface position is linear with a slope of 0.25 and average height of 0.3 . The lighter liquid is on the top of the heavier one and the gravity is 0.294 (all in consistent units). The geometry and the eight-noded finite element mesh used are shown in Fig. 3. The normal velocity is prescribed to zero in all faces of the tank while the tangential component is set to zero at the top and bottom

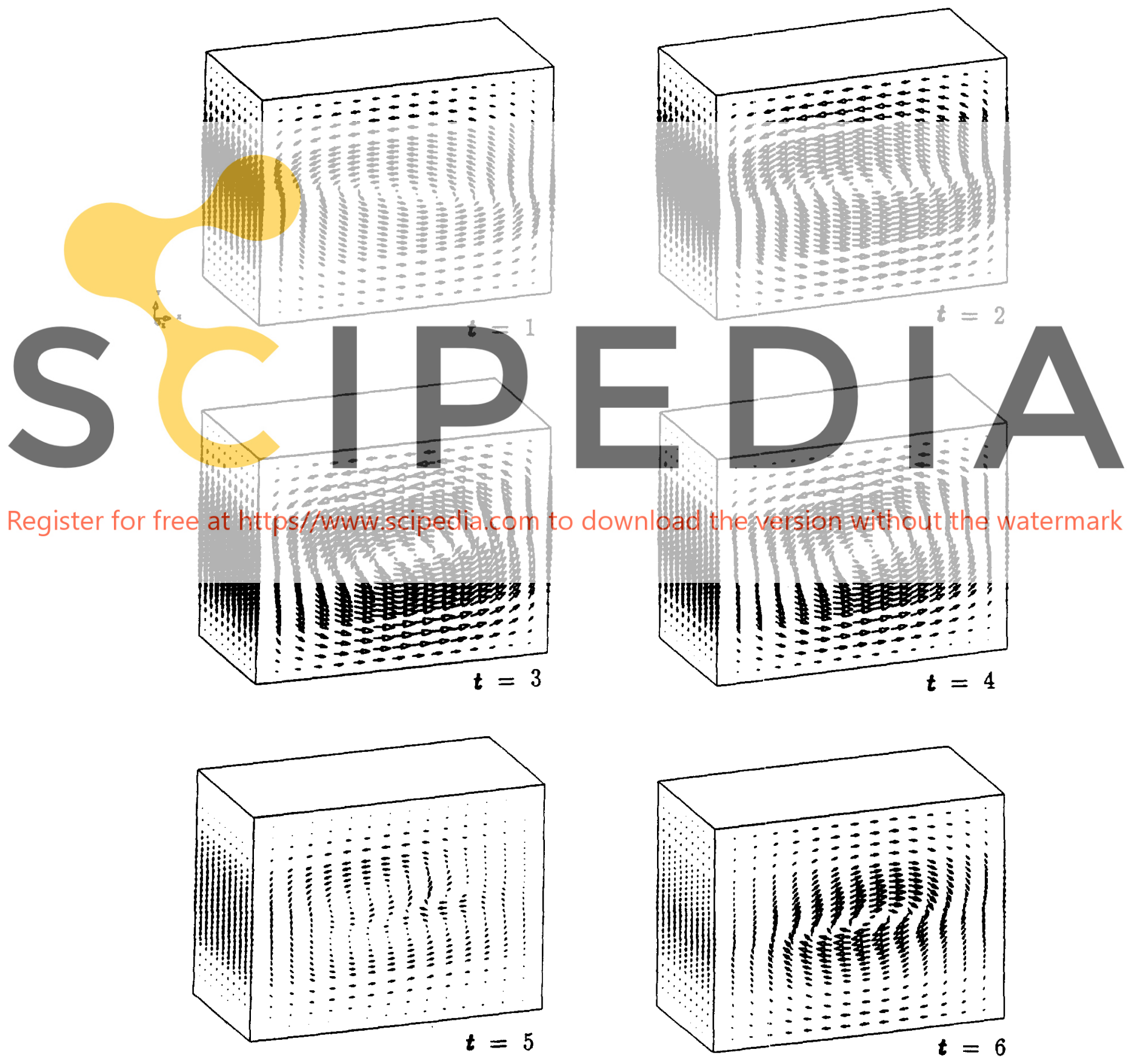

Fig. 5. Two-liquid interface problem in 3D. Velocity vectors at different time steps. 
faces. The pressure is taken equal to zero at the top right line. The interface position is obtained using the methodology developed in [13], consisting in tracking the interface by means of an arbitrary Lagrangian mesh using the total velocity of the fluid particles belonging to it. The transient analysis has been done using the time step considered in the 2D case $(0.5)[2,3,20]$. The 3D mesh in the $X-Y$ plane is more coarse than the mesh used in the $2 \mathrm{D}$ analysis $[2,3,20]$. The interface position, the velocity vectors and the isopressure planes at different time steps are plotted in Figs. 4, 5 and 6, respectively. A good approximation to the 2D results $[2,3,20]$ is obtained.
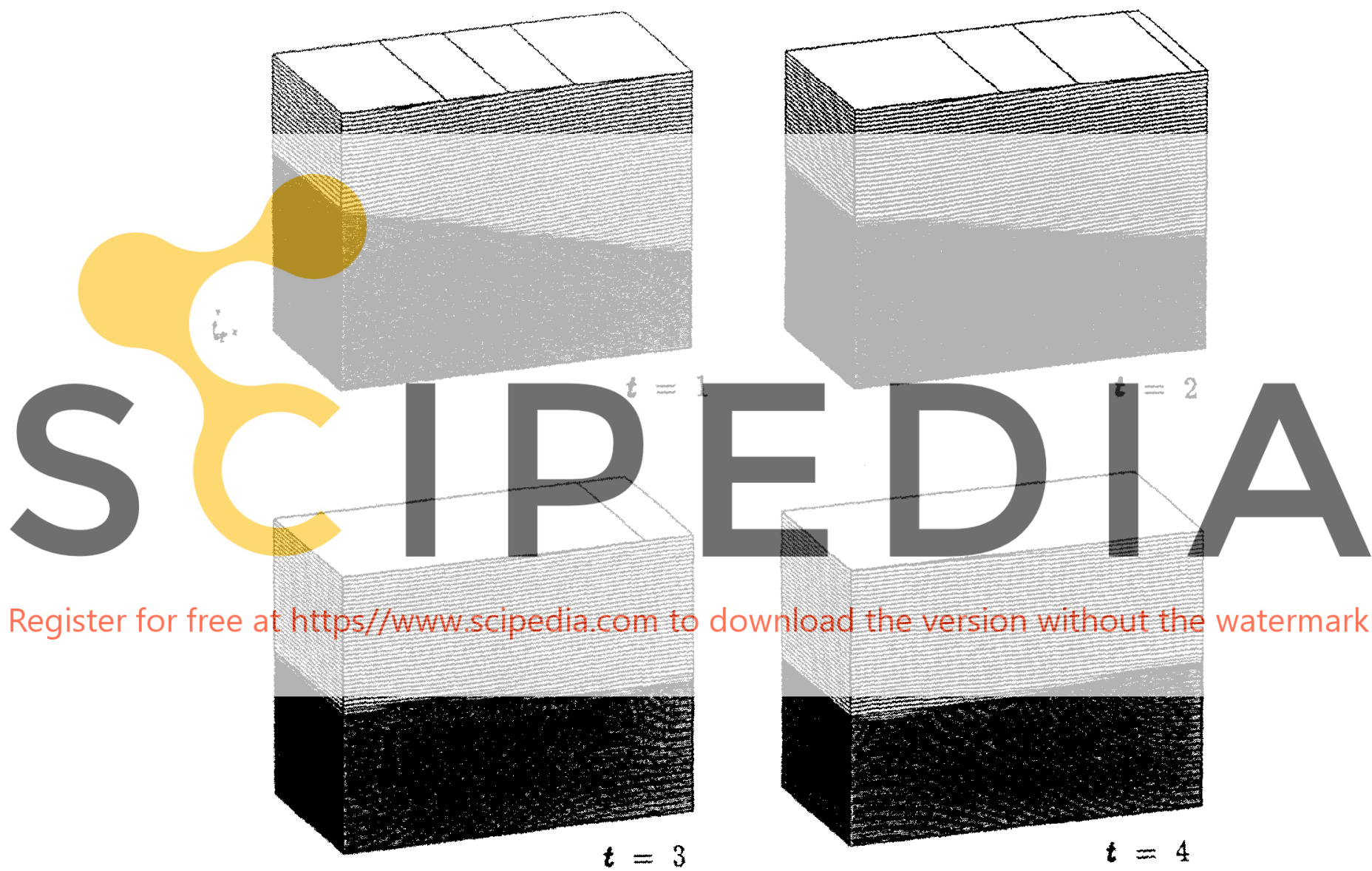

Register for free at https//wwscipedia.com to
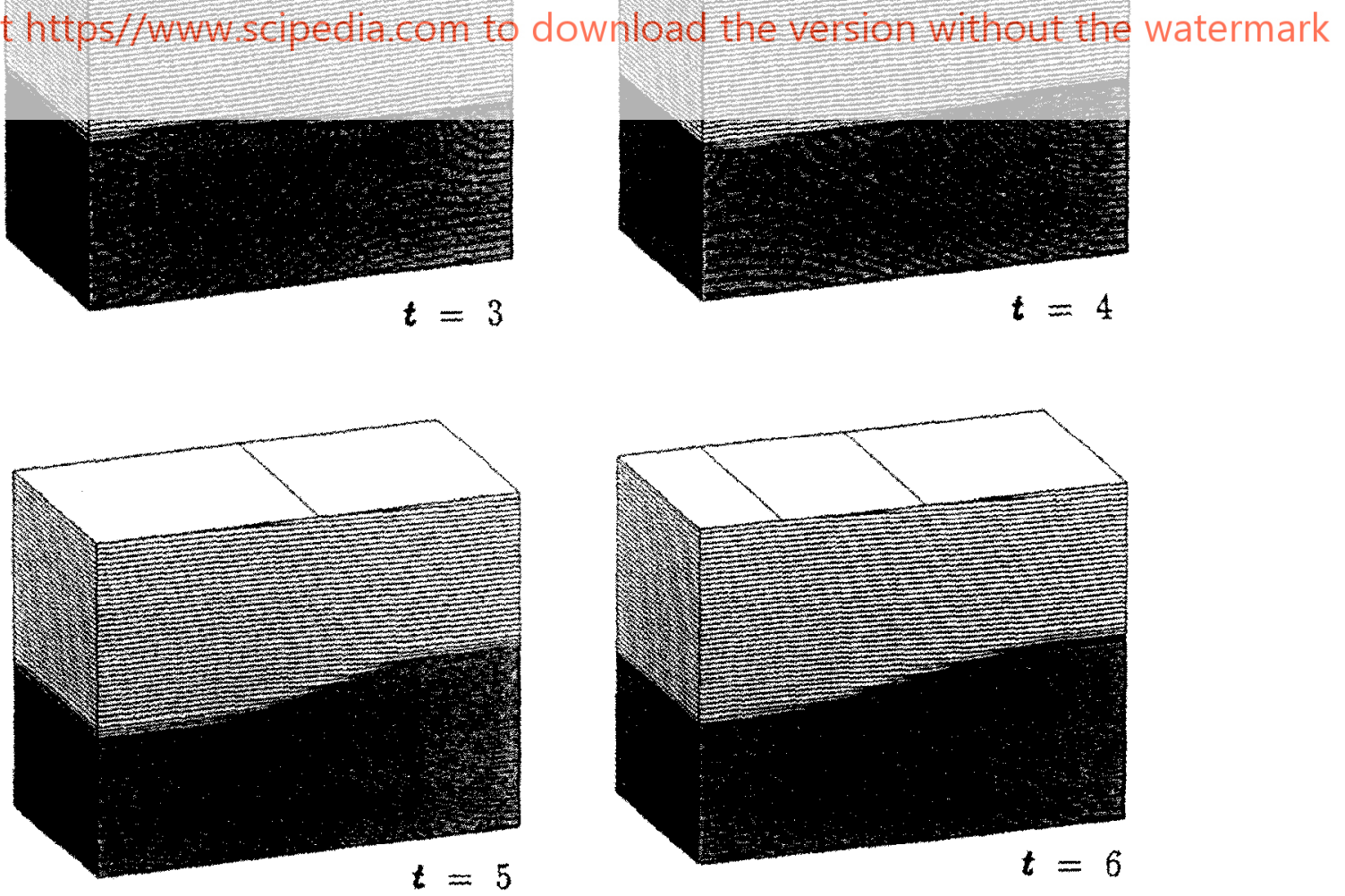

Fig. 6. Two-liquid interface problem in 3D. Isopressure planes at different time steps. 


\subsection{Solitary wave problem}

The propagation of a solitary wave over a constant depth has been studied by several authors [21,22]. It is a very interesting test in order to verify the temporal behaviour of the numerical algorithm proposed. In [21,22] the numerical solution is computed over a unique medium. The method used in the present work to track the moving surface [13] needs the definition of two media to identify the interface position. This fact adds additional difficulties to the problem. However, this problem description is more realistic if the two media involved in a physical wave propagation want to be considered. Fig. 7 shows the geometry used in the analysis. The sliding condition is assumed over the walls and the velocity is prescribed in the left side (inlet face) as $[3,21]$ :

$$
u_{x}=\frac{\eta c}{D}\left[\operatorname{sech}\left(\frac{c k}{D} t-4\right)\right]^{2}
$$

where: $c=[g(D+\eta)]^{1 / 2}$ and $k=[3 \eta / 4 D]^{1 / 2}$ [21]. The values for $g$ and $\eta$ are 1.0 and 0.86 , respectively [21]. The exit velocity for the upper fluid in the outlet face is neglected when its value are nearly zero (exactly when the wave have been completely formed). The transient analysis is done taking a time step of 1.7888 [21] and it is stopped before the wave reaches the right side. Two different sets of properties are analized considering that the fluid is inviscid in the original problem [21]. The results obtained with properties $\rho_{1}=10^{-6}, \mu_{1}=10^{-4}$, $\rho_{2}=1$ and $\mu_{2}=10^{-4}$ (case A) are shown in Figs. 8 and 9. The wave positions at different time steps are plotted in Fig. 8. The pressure history along the vertical line $x=L / 2$ is presented in Fig. 9. In this figure the wave path over the section $L / 2$ is registrated as a pressure increment. The wave positions at different time steps when the analysis is done with $\mu_{2}=0$ (case B) (the lower fluid is inviscid) are plotted in Fig. 10. The results published in

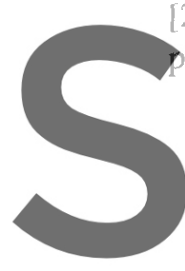
[21] and [22] are shown in

\section{osition at different times}
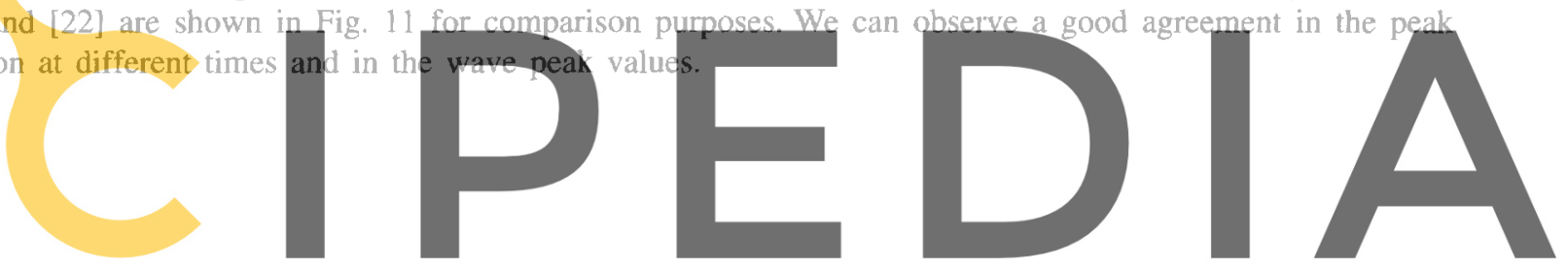

Register for free at https//www.scipedia.com to download- the vessipgn wigthout the watermark

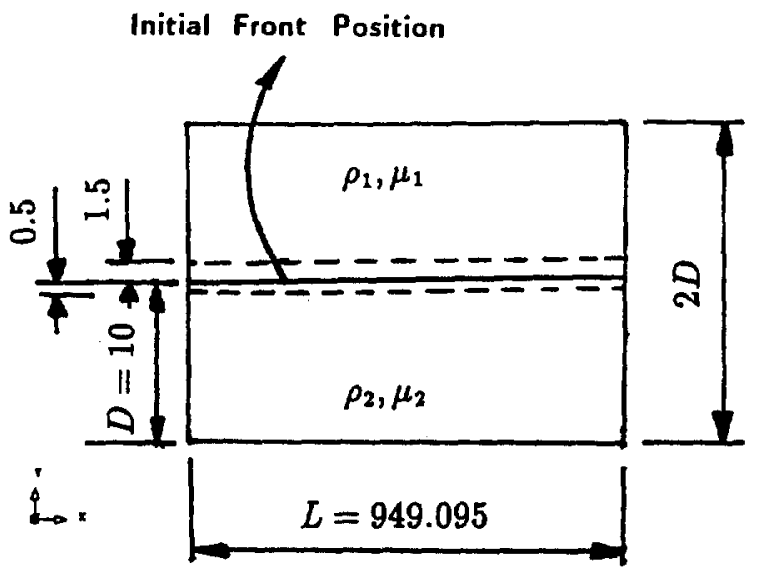

Fig. 7. Solitary wave problem. Geometry.

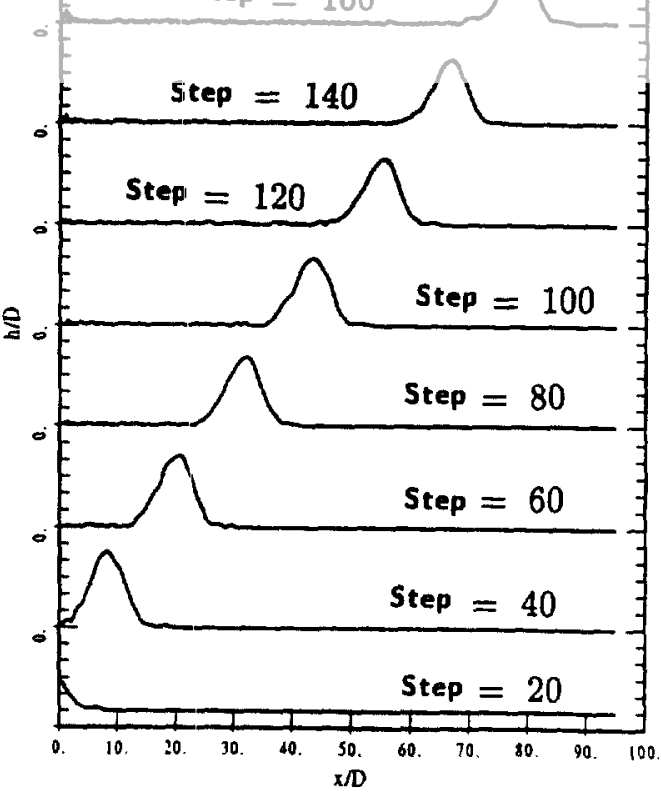

Fig. 8. Solitary wave problem. Wave position at different time steps (present work-Case A). 


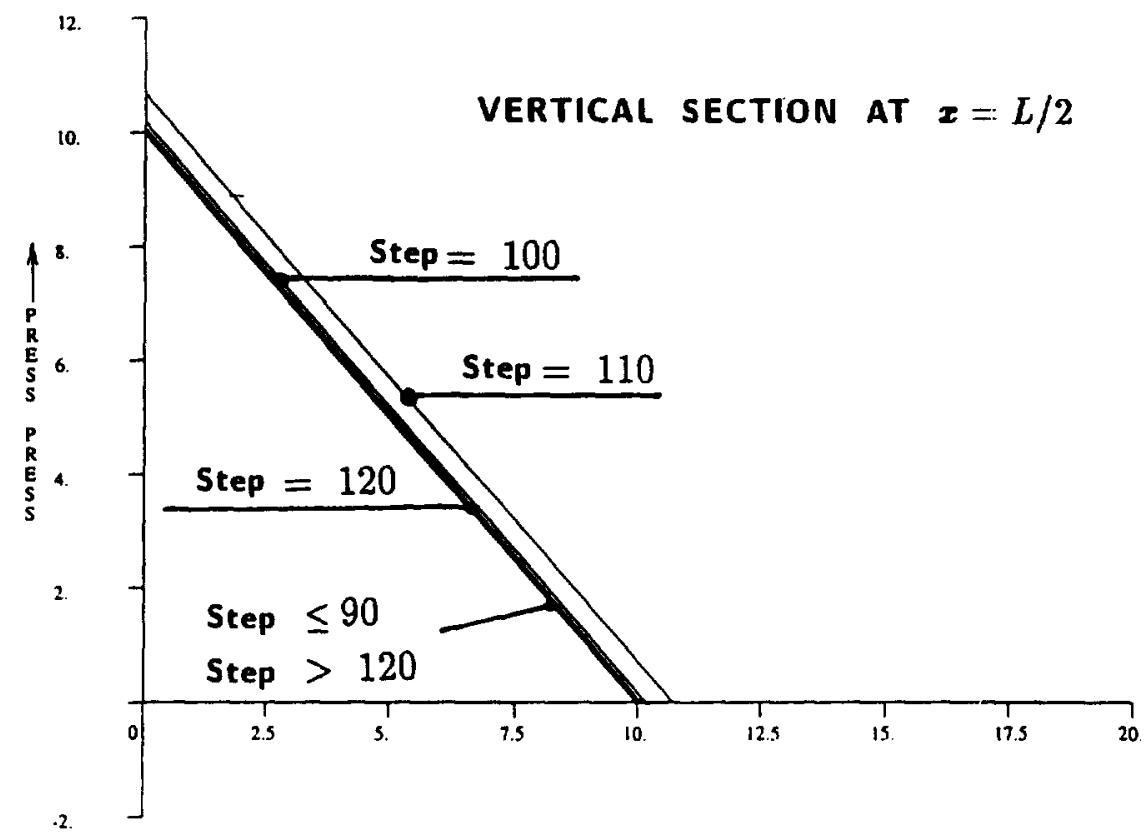

DISTANCE $\longrightarrow$

Fig. 9. Solitary wave problem. Pressure in section $x=L / 2$ at different time steps (present work-case A).

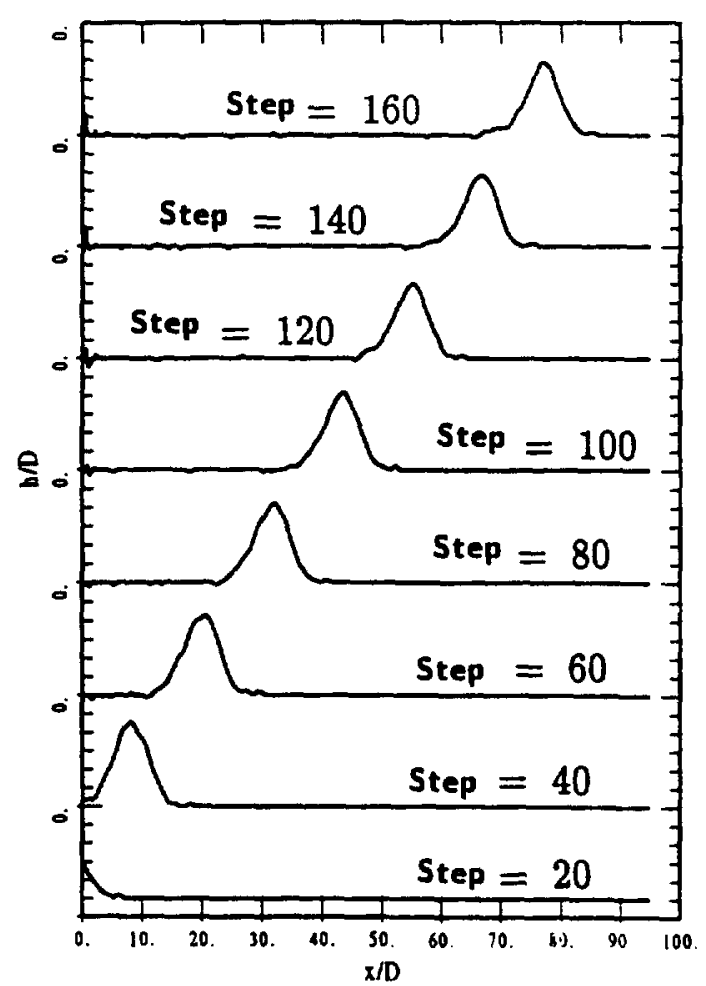

Fig. 10. Solitary wave problem. Wave position at different time steps (present work-Case B). 

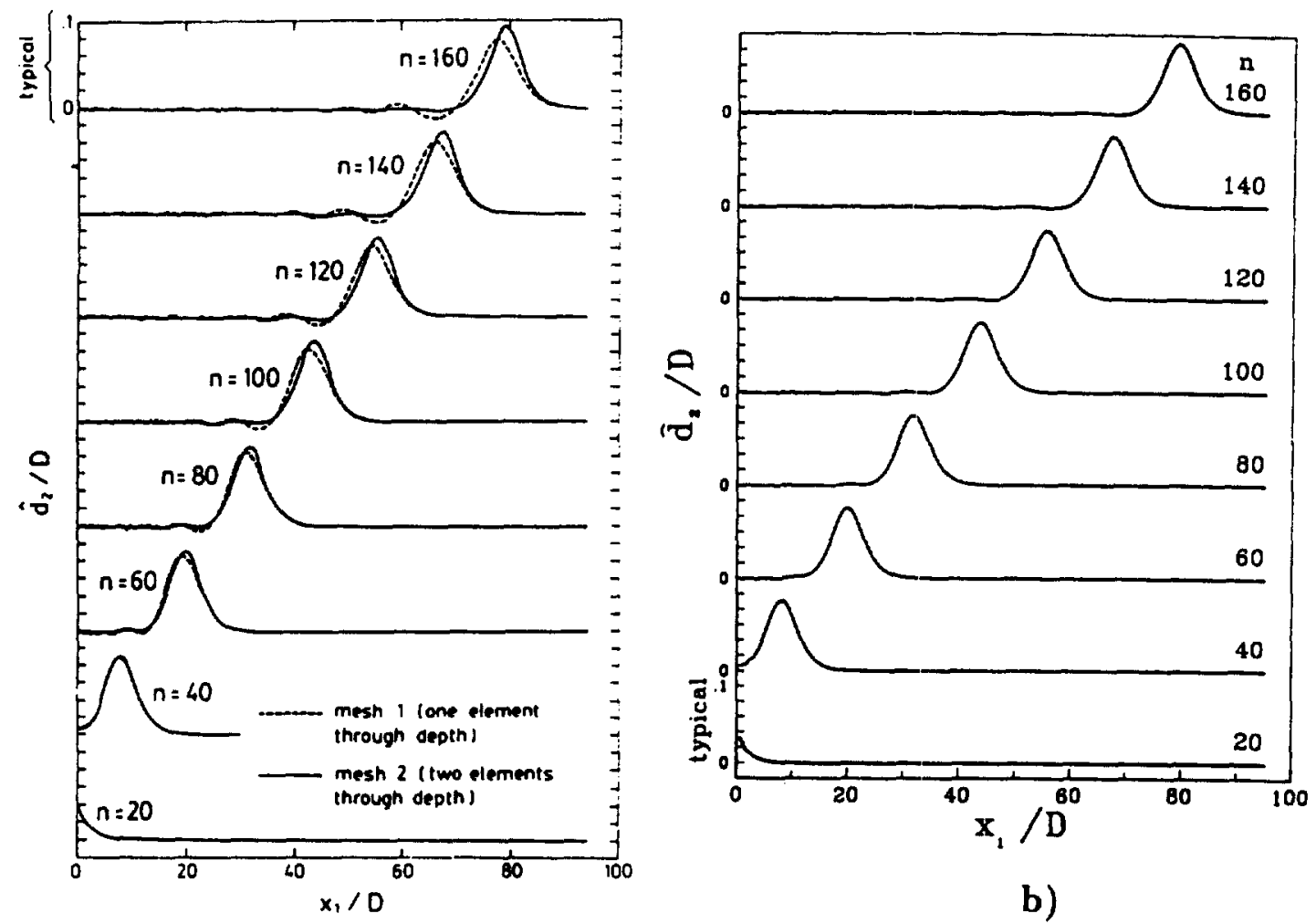

\section{a)}

Fig. 11. Solitary wave problem. Wave position at different time steps. (a) Hughes et al. [22]; (b) Huerta et al. [21].

\subsection{Sloshing problem}

The well-known practical application of this problem, for example the sloshing response of a liquid confined in a rigid-wall recipient or the overflow liquid during an earthquake, and the difficulties in the numerical modelization make of it a very challenging test for flows including a moving surface [20,21]. A closed tank filled with two liquids (Fig. 12) of properties: $\rho_{1}=10^{-6}, \mu_{1}=0.0, \rho_{2}=1.0$ and $\mu_{2}=0.002$, initially in rest is

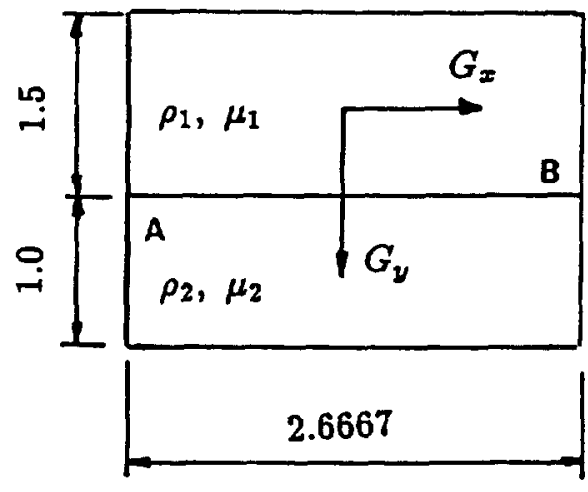

a)

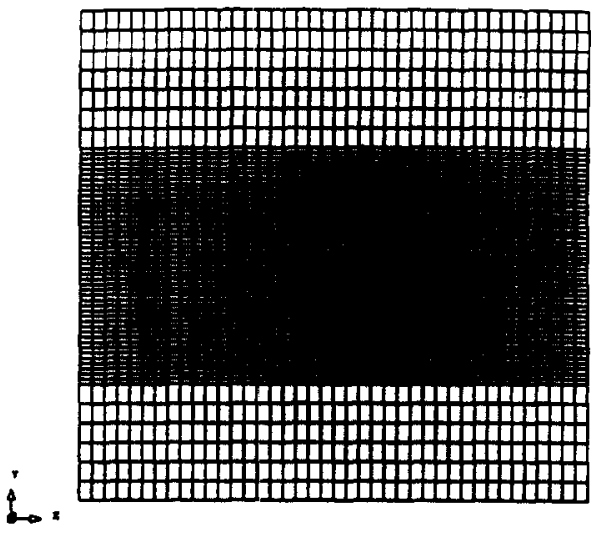

b)

Fig. 12. Sloshing problem. (a) Geometry; (b) finite element mesh. 


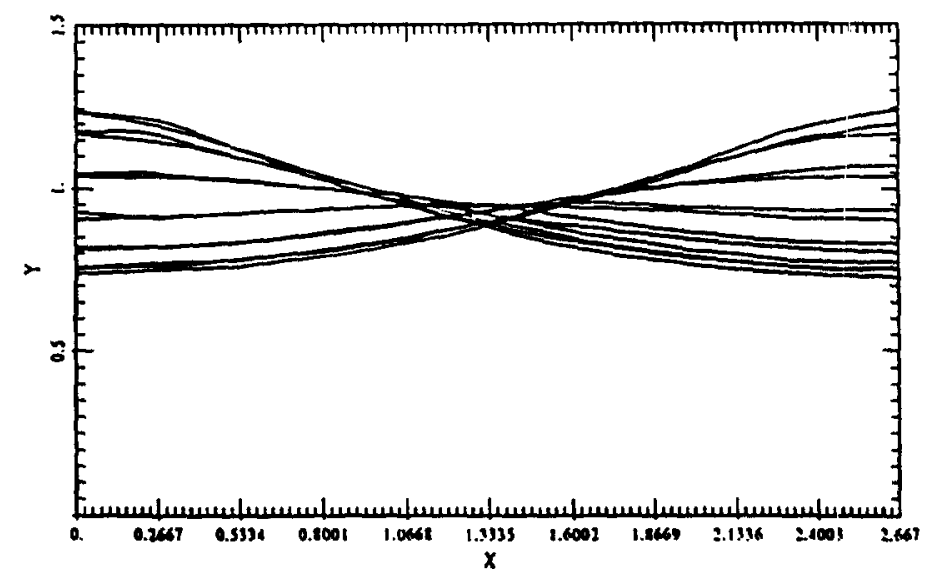

Fig. 13. Sloshing problem. Interface position at different time steps during the tenth cycle of charge.

considered for the numerical analysis of the problem. It is subjected to the gravity action $\left(G_{v}=-1.0\right)$ and to horizontal cyclical forces of the type $G_{x}=0.01 \sin (0.978 t)[20,21]$. The mesh discretization consist of 2320 four-noded elements (see (Fig. 12). A time step of 0.107 equivalent to 60 time steps per cycle is used in the transient analysis [21]. The interface positions at different time steps of the tenth cycle are plotted in Fig. 13. It is interesting to note that the vertical motion of the middle point of the interface approaches its physical behaviour [21]. The time-history positions of the interface contact points with the wall tank at the left and right sides (points A and B in Fig. 12) are presented in Fig. 14. A relative length $H_{r}=(H-1.0) / 1.0$ ) is plotted in this figure. This diagram shows a cualitative agreement with that presented in [20].

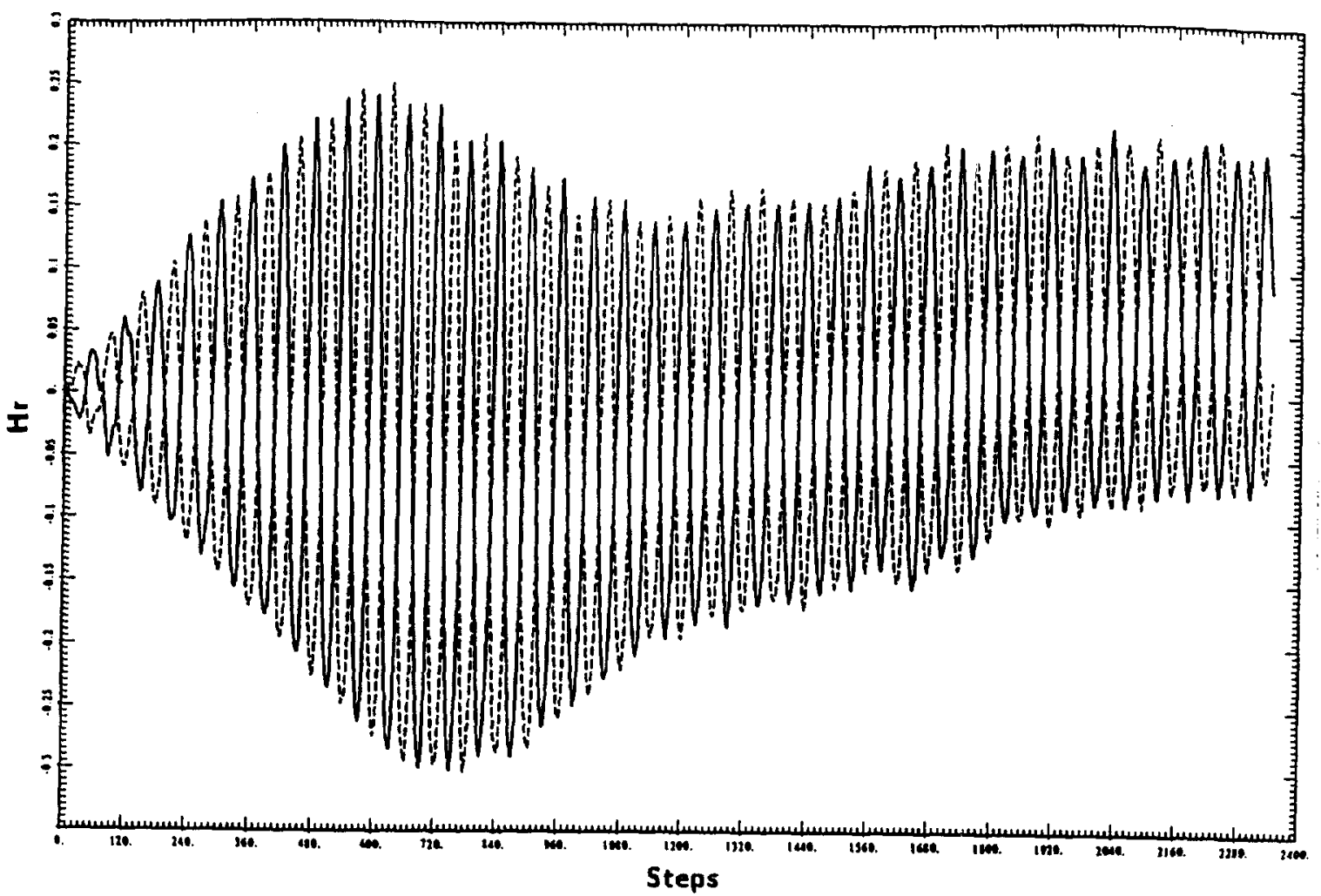

Fig. 14. Sloshing problem. Time-history position for points A and B (Fig. 1). Side A:-; side B:---. 


\section{Conclusions}

A generalized streamline finite element formulation for solving the incompressible Navier-Stokes flow equations has been presented. In this context, the element matrices have been explicitly defined. In this methodology the upwinding tensor does not require input tuning parameters and the velocity and pressure are interpolated using equal polinomial functions. Besides, the penalization methods are not needed in order to satisfy the imcompressibility condition.

Particular emphasis has been done in the tridimensional numerical solution of the Navier-Stokes equations and in the analysis of moving interface problems. The numerical examples show a good agreement between the results obtained using the present formulation with other numerical results reported by different authors.

The methodology proposed presents a very good behaviour in structured and unstructured meshes, as well as in coarse meshes. Moreover, the results computed with four-noded or three-noded 2D-elements are in a good agreement between them. A very good numerical performance can be observed in moderated and high Reynolds' number problems. Besides, the Stokes and low Reynolds' numbers problems present a very good behaviour too.

On the other hand, in moving surface problems, the refined mesh, required in the zone where the front is expected to move, is a limitation in the choice of the meshes. However, the meshes used in the numerical examples are similar to those proposed by other authors and the time steps are exactly those reported in the literature.

\section{Acknowledgments}

The first author thanks Antonio Huerta and Juan José Egózcue for useful discussions related to some topics of the sloshing problem presented.

\section{References}

[1] M.A. Cruchaga and E. Onate, A finite element formulation for incompressible flow problems using a generalized streamline operator, Comput. Methods Appl. Mech. Engrg. 143 (1997) 49-67.

[2] M.A. Cruchaga and E. Oñate, A finite element formulation for incompressible flow with moving surfaces using a generalized streamline operator, Proc. Ninth Int. Conf. on Finite Elements in fluids, Venezia, October 1995.

[3] M.A. Cruchaga, A incompressible flow model for moving surfaces problems, Doctoral Thesis, June 1996.

[4] T.J.R. Hughes and M. Mallet, A new finite element formulation for computational fluid dynamics: III. The generalized streamline operator for multidimensional advective-diffusive systems, Comput. Methods Appl. Mech. Engrg. 58 (1986) 305-328.

[5] T.J.R. Hughes, L.P. France and M. Balestra, A new finite element formulation for computational fluid dynamics: V. Circumventing the Babuska-Brezzi condition: a stable Petrov-Galerkin formulation of the stokes problem accomodating equal-order interpolations, Comput. Methods Appl. Mech. Engrg. 59 (1986) 85-99.

[6] T.J.R. Hughes, L.P. Franca and G.M. Hulbert, A new finite element formulation for computational fluid dynamics: VIII. The Galerkin/least-squares method for advective-diffusive equations, Comput. Methods Appl. Engrg. 73 (1989) 173-189.

[7] O.C. Zienkiewicz and J. Wu, Incompressbility without tears-How to avoid restrictions of mixed formulation, Int. J. Numer. Methods Engrg. 32 (1991) 1189-1203.

[8] L.P. Franca and T.J.R. Hughes, Convergence analyses of Galerkin least-quares methods for symmetric advective-diffusive forms of the Stokes and incompressible Navier-Stokes equations, Comput. Methods Appl. Mech. Engrg. 105 (1993) 285-298.

[9] T.E. Tezduyar, M. Behr and J. Liou, A new strategy for finite element computations involving moving boundaries and interfaces-The deforming-spatial-domain/space-time procedure: I. The concept and the preliminary numerical tests, Comput. Methods Appl. Mech. Engrg. 94 (1992) 339-351.

[10] P.A.B. De Sampaio, A Petrov-Galerkin formulation for the incompressible Navier-Stokes equations using equal order interpolation for velocity and pressure, Int. J. Numer. Methods Engrg. 31 (1991) $1135-1149$.

[11] M. Storti, N. Nigro and S. Idelsohn, Stabilizing equal-order interpolations for mixed formulations of Navier-Stokes equations via SUPG method, Internal report.

[12] G. Hauke and T.J.R. Hughes, A unified approach to compressible and incompressible flows, Comput. Methods Appl. Mech. Engrg. 113 (1994) 389-395.

[13] M. Cruchaga, E. Onate and S. Idelsohn, On the pseudomaterial approach for the analysis of transient forming processes, Comm Numer. Methods Engrg. 11 (1995) 137-148.

[14] O.C. Zienkiewicz and R.L. Taylor, The Finite Element Method 4th edition (McGraw-Hill Book Company). 
[15] J.R. Hughes and L.P. Franca, A new finite element formulation for computational fluid dynamics: VII. The Stokes problem with various well-posed boundary conditions: symmetric formulations that converge for all velocity/pressure spaces, Comput. Methods Appl. Mech. Engrg. 65 (1987) 85-96.

[16] C. Conca, C. Pares, O. Pironneau and M. Thiriet, Navier-Stokesequations with imposed pressure and velocity fluxes, Int. J. Numer. Methods Fluids 20 (1995) 267-287.

[17] M. Peeters, W. Habashi and B.Q. Nguyen, Finite element solutions of the incompressible Navier-Stokes equations by a Helmholtz velocity decomposition, Int. J. Numer. Methods Fluids, 13 (1991) 135-144.

[18] J. Solà-Morales Rubió, Conditions au bord sur la vitesse et la pression pour les équations de Navier-Stokes dans une conduit finite, C.R. Acad. Sc. Paris, t.299, Série 1, no11 (1984).

[19] G. Guj and F. Stella, A vorticity-velocity method for the numerical solution of 3D incompressible flow, J. Comput. Phys. 106 (1993) $286-298$.

[20] T.E. Tezduyar, M. Behr and J. Liou, A new strategy for finite element computations involving moving boundaries and interfaces-The deforming-spatial-domain/space-time procedure: II. Computation of free-surface flows, two-liquid flows, and flows with drifting cylinders, Comput. Methods Appl. Mech. Engrg. 94 (1992) 339-351.

[21] A. Huerta and W.K. Liu, Viscous flow with large free surface motion, Comput. Methods Appl. Mech. Engrg, 69 (1988) $277-324$.

[22] T.J.R. Hughes, W.K. Liu and T.K. Zimmerman, Lagrangian-Eulerian finite element formulation for incompressible viscous flows, Comput. Methods Appl. Mech. Engrg. 29 (1981) 329-349. 